\title{
Childhood Perioral Dermatitis - Challenging Treatment
}

\author{
Anca Chiriac ${ }^{1,2,3}$, Adriana Diaconeasa ${ }^{4}$, Cristian Podoleanu ${ }^{5}$, Simona Stolnicu ${ }^{6,7}$ \\ 1 Nicolina Medical Center, Department of Dermatology, Iași, Romania \\ 2 Apollonia University, Iași, Romania \\ 3 P. Poni Research Institute, Romanian Academy, Iași, Romania \\ 4 Dermatology Outpatient Care Center, "Grigore Alexandrescu" Clinical Emergency Hospital for Children, Bucharest, Romania \\ 5 Department of Internal Medicine, University of Medicine and Pharmacy, Tîrgu Mureș, Romania \\ ${ }^{6}$ Department of Pathology, University of Medicine and Pharmacy, Tîrgu Mureș, Romania \\ 7 Histopat Invest Laboratory, Tîrgu Mureş, Romania
}

\section{CORRESPONDENCE}

\section{Cristian Podoleanu}

Str. Gheorghe Marinescu nr. 38

540139 Tîrgu Mureş, Romania

Tel: +40 265215551

E-mail: podoleanu@me.com

\section{ARTICLE HISTORY}

Received: November 15, 2017

Accepted: January 20, 2018
Anca Chiriac • Str. Hatman Sendrea nr. 2, 700613 lași, Romania. Tel: +40332808703

Adriana Diaconeasa • Bld. lancu de Hunedoara nr. 30, 011743 Bucharest, Romania. Tel: +40 213169366

Simona Stolnicu • Str. Gheorghe Marinescu nr. 38 540139 Tîrgu Mureș, Romania. Tel: +40 265215551

\section{ABSTRACT}

Background: Also known as papulopustular dermatitis (chronic form), rosacea-like dermatitis, periorificial dermatitis, or airhostess' dermatitis, perioral dermatitis is a commonly encountered dermatological disease, especially in adult women and less frequently in children. Its diagnosis and treatment are a challenge especially in small children. Case report: We present the case of a 2-year-old girl referred to the dermatologist for widespread erythematous papules, vesicles, and pustules on the perioral area, nasolabial folds, and on the outer region of the lower eyelids. Several diagnoses had been established during the previous months: impetigo, atopic dermatitis, seborrheic dermatitis, allergic contact dermatitis, childhood rosacea, demodecidosis, infantile acne, and variable therapeutic approaches had been tried, with no clinical improvement. Clarithromycin $250 \mathrm{mg} /$ day orally associated with the application of $2 \%$ erythromycin solution were successfully used. Conclusion: This case highlights the importance of the clinical diagnosis of perioral dermatitis in young children, of excluding other diagnoses and worthless treatments, and also the chronic evolution of the disease and its individualized treatment.

Keywords: perioral, dermatitis, macrolides, case report

\section{BACKGROUND}

Also known as papulopustular dermatitis (chronic form), rosacea-like dermatitis, periorificial dermatitis, or airhostess' dermatitis, ${ }^{1}$ perioral dermatitis is a commonly encountered dermatological disease, especially in adult women and less frequently in children. ${ }^{2,3}$ Its diagnosis and treatment are a challenge especially in small children.

\section{CASE PRESENTATION}

A 2-year-old girl was referred to the dermatologist for widespread erythematous 


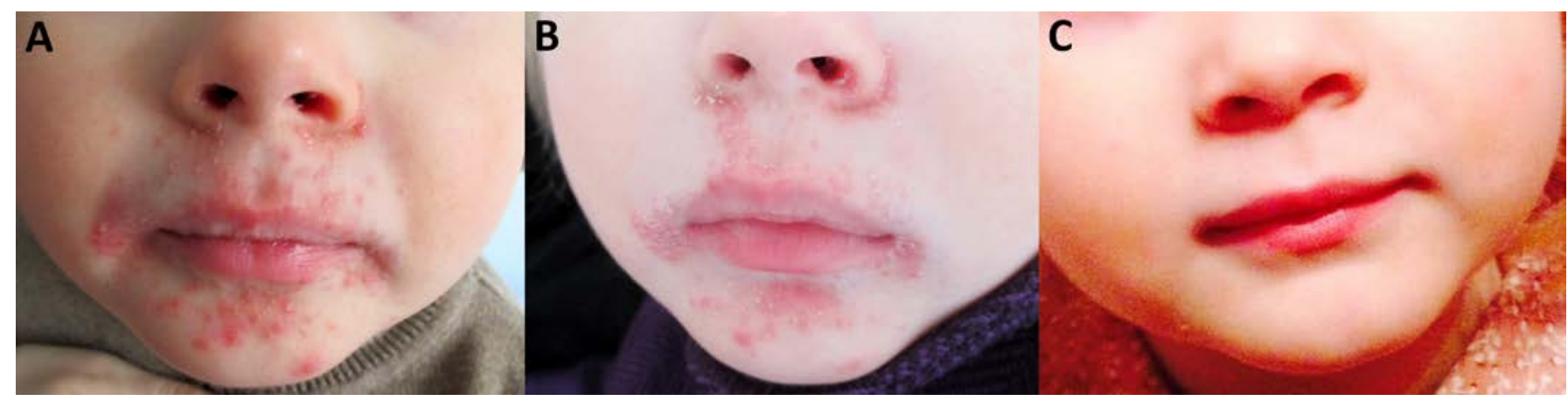

FIGURE 1. A - Perioral dermatitis diagnosed at the admission in the hospital. B - Clinical picture after 2 weeks of treatment: no pustules, papules or vesicles; desquamation and erythema; lesion of the inferior eyelids almost cleared. $\mathbf{C}-$ Clinical aspect after 4 weeks of treatment.

papules, vesicles and pustules on the perioral area, nasolabial folds, and on the outer region of the lower eyelids (Figure 1A). She was an otherwise healthy child, with no allergic background. She had developed the skin lesions progressively within a month, and her mother reported that the child complained about a burning sensation, but not itching. Several diagnoses had been established during the previous months: impetigo, atopic dermatitis, seborrheic dermatitis, allergic contact dermatitis, childhood rosacea, demodecidosis, infantile acne, and variable therapeutic approaches had been tried, with no clinical improvement, but resulting in anxiety for the family members. Emollients, topical steroids, topical antibiotics (metronidazole $0.5 \%$, fusidic acid, tetracycline ointment), and pimecrolimus had been applied once or twice daily on the affected areas, but with no results. The patient had been treated with oral antihistamines for 3 months and even underwent a short course of systemic steroids.

Meticulous laboratory investigations have been carried out, including an allergic panel and specific IgE, searching for Demodex folicullorum as well as allergy consultations, but all proved to be within normal limits.

Based on the clinical picture, the clinical diagnosis of a severe form of perioral dermatitis (PODSI - perioral dermatitis severity index -7 ) in a young child was presumed. Discontinuation of all topical steroid creams, cosmetics, fluoride toothpaste, or other topical medication was the first recommendation. Psychological support was recommended for the very anxious mother, reassuring her about the benignity and favorable evolution of the disease and the lack of risk of scarring or other marks on the girl's face.

TABLE 1. Differential diagnosis of perioral dermatitis in children

\begin{tabular}{ll}
\hline Frequent diseases & Hints to differentiate from perioral dermatitis \\
\hline Rosacea (Figure 2A) & - rare in children \\
& is a central-facial disease: chin, nose, cheeks \\
& no lesions around the mouth or eyes, although ocular involvement \\
& is frequently seen \\
& erythema, papulopustules (without comedones), telangiectasia \\
& - mostly in infants during the first three months of life \\
& - slight predominance in boys \\
Seborrheic dermatitis (Figure 2B) & thick, greasy scales \\
& location of the lesions: scalp border, scalp, eyebrows, retroauricu- \\
& lar and nasolabial areas \\
Atopic dermatitis (Figure 2C, 2D) & seborrheic blepharitis can be present \\
& very rare papules, pustules \\
- other signs of atopy or/and atopic dermatitis are noticed & pruritus present and intense \\
Infantile acne (Figure 2E) & chronic evolution with flares \\
Contact eczema of the face (Figure 2F) & pustules, papules, comedones, cysts, small scars, nodules \\
& different location of lesions \\
& erythematous vesicles and papules \\
& pruritus \\
\hline
\end{tabular}




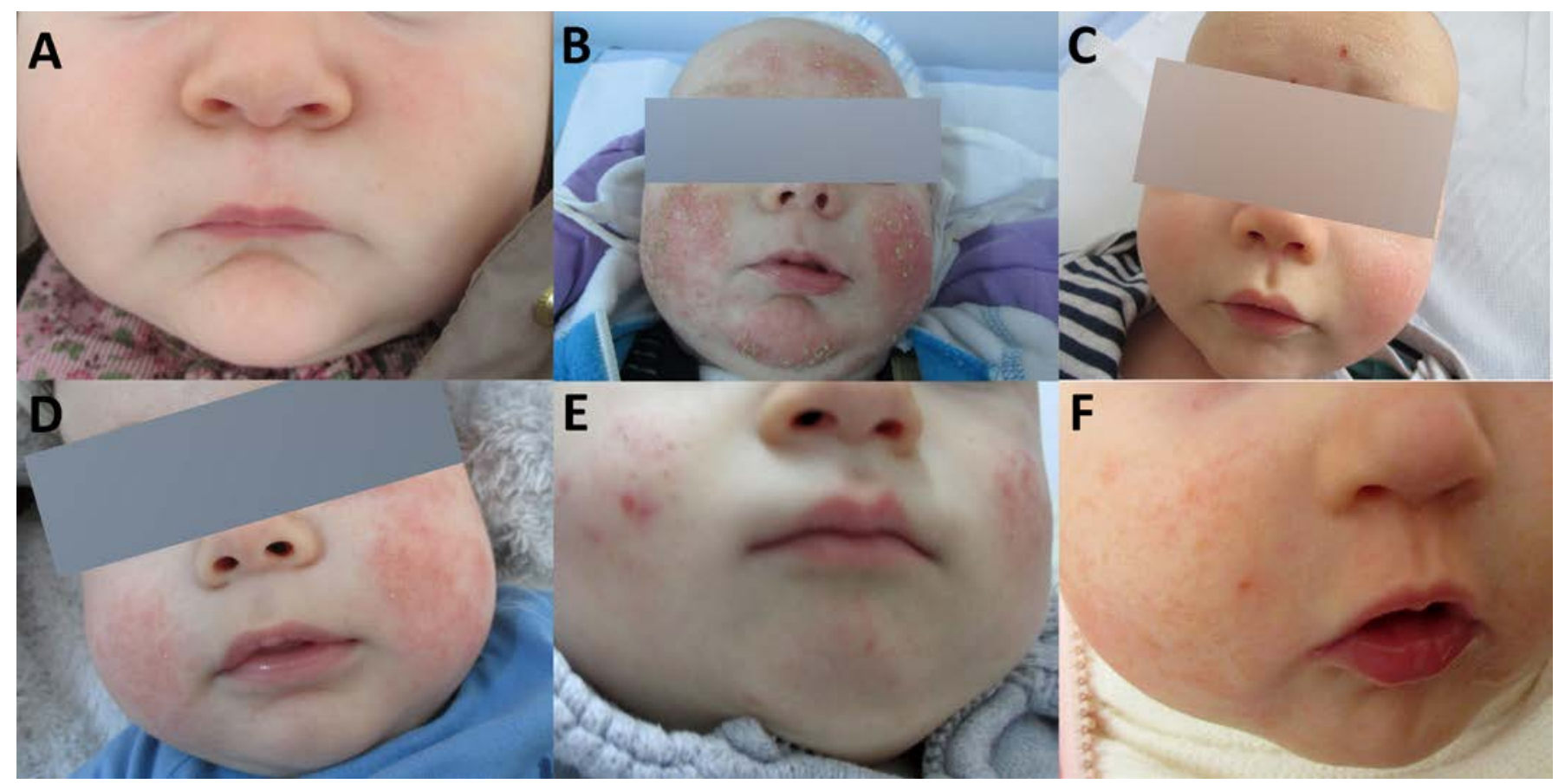

FIGURE 2. A - Rosacea in a young child: flash over the cheeks, chin, and frontal area. B - Seborrheic dermatitis in a young child: yellow crusty greasy scales and intense erythema on the face. C - Atopic dermatitis: xerosis and scratch marks on the forehead. D - Atopic dermatitis: dry and scaly patches on the cheeks, discrete periocular erythema. E - Infantile acne: nodules, pits, open comedones and scars on the cheeks. F - Contact eczema of the face: erythema, pin papules, and fine desquamation on the entire face.

Due to the young age of the patient, tetracyclines were forbidden, and clarithromycin $250 \mathrm{mg}$ /day orally associated with the application of $2 \%$ erythromycin solution were recommended. The treatment was continued for 4 weeks, and positive results were seen after one month of therapy (Figure 1B, C). Informed consent to publish the case was obtained from the parents of the pediatric patient.

\section{DISCUSSIONS}

The diagnosis of perioral dermatitis is established mostly on clinical grounds. Clinical features are represented by erythema, papules, vesicles and pustules, sometimes associated with fine scaling, symmetrically distributed in the perioral area, but not on the lips. In severe chronic forms, typical lesions are widespread in the nasolabial folds, on the chin, glabella, forehead, and eyelids. Lesions on the periocular area are typically localized on the outer area of the lower eyelids. The evolution of the disease is chronic, with flares and partial remissions, accompanied by burning or stinging sensations and rarely by pruritus. Facial edema is rare in children.

Based on the intensity of the erythema, papules, and scaling, a severity grade system named PODSI, ${ }^{4}$ which can be used for monitoring the patient, was established. PODSI is based on lesion quantification: erythema: grade 0-3; papules: $0-3$; scaling: $0-3$. The interpretation is quite simple: PODSI $0.5-2.5=$ mild; $3-5.5=$ moderate $; 6-9=$ severe.

In current practice, especially in young children where biopsy is rarely performed, particularly on the face, no specific laboratory work-up is necessary. The diagnosis is almost purely clinical; searching for Demodex is quite difficult and very rarely positive, although perioral dermatitis is considered by some authors to be a form of rosacea in children.

The differential diagnosis of perioral dermatitis in children is also based on clinical grounds, taking into consideration particularities related to the age of the patient (Table 1, Figure 2).

The treatment of perioral dermatitis in young children is based on the severity index of the disease, previous treatments, compliance and adherence to the therapeutic scheme, surveillance and monitoring the disease and the treatment.

The first step is to avoid or to discontinue any treatment and to closely follow-up the child. The next step is based on topical medication, especially topical antibiotics (metronidazole, erythromycin), azelaic acid (irritant effects on the skin, lack of compliance), pimecrolimus, tacrolimus. ${ }^{5-7}$ Systemic therapy, such as tetracyclines, isotretinoin, or dapsone, has a limited use in young children because of 
contraindications and adverse reactions. Macrolides, especially erythromycin and clarithromycin, remain an option in children. ${ }^{8}$

Perioral dermatitis is still an enigmatic disease, especially in young children, with an undefined pathogenic mechanism, without a diagnosis guideline and a standardized treatment.

Disputable trigger factors have been described as having a role in perioral dermatitis: topical or inhaled steroids, cosmetics, infectious agents (Fusiform bacteria, Candida spp, Demodex folliculorum), climatic offenders (wind, hot or cold weather, ultraviolet light), genetic predisposition or immunosuppression. ${ }^{1,3}$

\section{CONCLUSIONS}

This case highlights the importance of the clinical diagnosis of perioral dermatitis in young children, of excluding other diagnoses and worthless treatments, and also of the chronic evolution of the disease and individualized treatment. Systemically and topically administered macrolides proved to be efficient in this particular case of severe perioral dermatitis in a 2-year-old girl.

\section{CONFLICT OF INTEREST}

None for all authors.

\section{REFERENCES}

1. Lipozencic J, Liubojevic S. Perioral dermatitis. Clin Dermatol. 2011;29:157161.

2. Hafeez ZH. Perioral dermatitis: an update. Int J Dermatol. 2003;42:514-517.

3. Wollenberg A, Bieber T, Dirschka T. Perioral dermatitis. J Dtsch Dermatol Ges. 2011:9:422-427.

4. Wollenberg A, Oppel T. Scoring of skin lesions with the perioral dermatitis severity index. Acta Derm Venereol. 2006;86:254-255.

5. Jansen T. Azelaic acid as a new treatment for perioral dermatitis: results from an open study. Br J Dermatol. 2004;151:933-934.

6. Rodriguez-Martin M, Saez-Rodriguez M, Carnerero-Rodriguez A, et al. Treatment of perioral dermatitis with topical pimecrolimus. J Am Acad Dermatol. 2007;56:529-530

7. Gerber PA, Neuman NJ, Ruzicka T, Bruch-Gerhard D. Perioral dermatitis and treatment with tacrolimus. Hautarzt. 2005:56:967-968.

8. Choi YL, Lee KJ, Cho HJ, et al. Case of childhood granulomatous periorificial dermatitis in a Korean boy treated by oral erythromycin. $J$ Dermatol. 2006;33:806-808. 